\title{
THE VANISHING PROBLEM OF THE STRING CLASS WITH DEGREE 3
}

\section{KATSUHIKO KL'RIBAYASHI and TOSHIHIRO YAMAGUCHI}

\author{
(Recured 20 May 1997; revised 11 May 1998)
}

Communicated by J. A. Hillman

\begin{abstract}

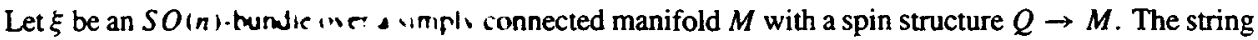
class is an obstructum I. lit itr :tru lure group $L \operatorname{Spin}(n)$ of the loop group bundle $L Q \rightarrow L M$ to the

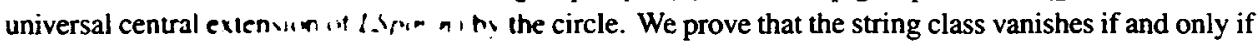

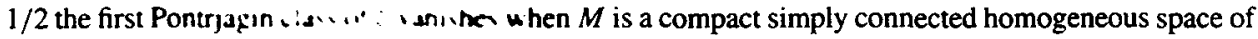
rank one, a simply comncird \& dinnumal manifold or a finite product space of those manifolds. This result is deduced hw unn: the l ikrerg l/core spectral sequence converging to the mod $p$ cohomology of $L M$ whose $E_{:}$-term I. the tha h n nith homology of the mod $p$ cohomology algebra of $M$. The key

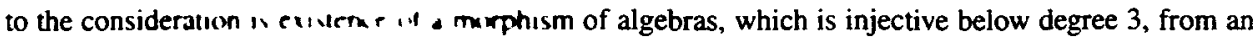
important graded commuts:ver a: :etrs into the Hochschild homology of a certain graded commutative algebra.
\end{abstract}

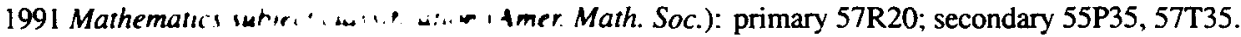

\section{Introduction}

Let $X$ be a simply connecied space and $L X$ the space of continuous closed paths on $X$. If $M$ is a simply connected manifold, then we regard $L M$ as the space of smooth free loops on $M$. Throughout this paper, the map $\int_{S^{1}}$ oe $v^{*}: H^{*}(X ; \mathbf{Z}) \rightarrow$ $H^{*-1}(L X ; Z)$ will be denoted by $\mathscr{D}_{X}$ and called the $D$-map of $X$, where $\int_{S^{1}}: H^{*}\left(S^{1} \times\right.$ $L X ; \mathbf{Z}) \rightarrow H^{*-1}(L X ; \mathbf{Z})$ is the integration map along $S^{1}$ and $e v: S^{1} \times L X \rightarrow X$ is the evaluation map. We say the $D$-map $\mathscr{D}_{X}$ is good if $\mathscr{D}_{X}: H^{4}(X ; \mathbf{Z}) \rightarrow H^{3}(L X ; \mathbf{Z})$ is a monomorphism.

Let $\xi$ be an $S O(n)$-bundle over a simply connected manifold $M$ with a spin structure $Q \rightarrow M$. In [8], McLaughlin defined the string class $\mu(Q)$ in $H^{3}(L X ; \mathbf{Z})$ which

(C)1998 Australian Mathematical Society 0263-6115/98\$A2.00+0.00 
is an obstruction to lift the structure group of the $L \operatorname{Spin}(n)$-bundle $L Q \rightarrow L M$ to $\widehat{L \operatorname{Spin}(n)}$, where $\mathbf{T} \rightarrow \widehat{L \operatorname{Spin}(n)} \rightarrow L \operatorname{Spin}(n)$ is the universal central extension of $L \operatorname{Spin}(n)$ by the circle and $n \geq 5$. It is asserted in [8, Lemma 2.2] that the first Pontrjagin class $p_{1}(\xi)$ is two times the pullback of the generator $\iota$ of $H^{4}(B \operatorname{Spin}(n) ; \mathrm{Z})$ by the classifying map of a spin structure $Q \rightarrow M$ for $\xi$. Following [8], we denote the pullback of $\iota$ by $\frac{1}{2} p_{1}(\xi)$. The argument of the proof of [8, Theorem 3.1] enables us to conclude that the $D$-map $\mathscr{D}_{M}: H^{4}(M ; \mathbf{Z}) \rightarrow H^{3}(L X ; \mathbf{Z})$ carries $\frac{1}{2} p_{1}(\xi)$ to the string class $\mu(Q)$. Therefore if the $D$-map of $M$ is good, then $\mu(Q)$ vanishes if and only if $\frac{1}{2} p_{1}(\xi)$ vanishes for any $S O(n)$-bundle $\xi$ with a spin structure $Q \rightarrow M$. In this case we can deduce that the $L \operatorname{Spin}(n)$-bundle over the infinite dimensional manifold $L M$ has a string structure if and only if $1 / 2$ the first Pontrjagin class of the $S O(n)$-bundle over the finite dimensional manifold $M$ vanishes. Our goal is to study which manifolds have a good $D$-map. In [8, Theorem 3.1], it has been proved that every 2 -connected manifold has a good $D$-map. Recently, Kuribayashi has proved

THEOREM A ([4, Theorem 1]). Let $M$ be a simply connected manifold. If $H^{4}(M ; \mathbf{Z})$ is torsion free and $\operatorname{dim} H^{2}(M ; \mathbf{R}) \leq 1$. Then the $D$-map of $M$ is good. Therefore, in this case, $\frac{1}{2} p_{1}(\xi)$ vanishes if the string class $\mu(Q)$ vanishes.

We can deduce from Theorem A that the complex Grassmann manifold has a good $D$-map.

In this paper, the following theorem will be proved. As a consequence we can obtain many manifolds whose $D$-maps are good.

THEOREM 1.1. Let $X$ be a simply connected space. Suppose that (1.1) $H^{4}(X ; \mathbf{Z}) \cong \mathbf{Z} \oplus \cdots \oplus \mathbf{Z} \oplus \mathbf{Z} / p_{1} \oplus \cdots \mathbf{Z} / p_{k}$, where $p_{i}$ is prime for any $i$, and (1.2) $x^{2}=0$ for any element $x \in H^{2}(X ; \mathbf{Z} / 2)$ if $H^{4}(X ; Z)$ has 2-torsion.

Then the D-map of $X$ is good.

Theorem 1.1 is a generalization of Theorem A. Applying Theorem 1.1 to simply connected 4-manifolds and compact, simply connected homogeneous spaces of rank one, we have

THEOREM 1.2. Let $M$ be a simply connected 4-dimensional manifold, compact simply connected homogeneous spaces of rank one or a finite product of those manifolds. Then the D-map of $M$ is good. Therefore the string class $\mu(Q)$ vanishes if and only if $\frac{1}{2} p_{1}(\xi)$ vanishes.

The classification of compact, simply connected, homogeneous space of rank one has been made by Oniscik [9]. In [7], McCleary and Ziller have determined the $\bmod p$ cohomology of the homogeneous spaces completely for any prime $p$. These results are also used to prove Theorem 1.2. Following [7, p. 767], we now list such homogeneous spaces which are not diffeomorphic to spheres or projective spaces: 
(1) $(S O(2 n+1), S O(2 n-1) \times S O(2), 1)$,

(2) $(S O(2 n+1), S O(2 n-1), 1)$

(3) $(S U(3), S O(3), 4)$,

(4) $(S p(2), S U(2), 10)$,

(5) $\left(G_{2}, S O(4),(1,3)\right)$,

(6) $\left(G_{2}, U(2), 3\right)$,

(7) $\left(G_{2}, S U(2), 3\right)$,

(8) $\left(G_{2}, S O(3), 4\right)$,

(9) $\left(G_{2}, S O(3), 28\right)$,

where the triple $(G, H, i)$ consisting of the Lie group $G$, the subgroup $H$ and the integer or a pair of integers means the homogeneous space $G / H$ of $G$ by the subgroup $H$ with the index $i$. Here the index of the subgroup $H$ of $G$ is that of the subalgebra $\operatorname{Lie}(H)$ of the Lie algebra Lie $(G)$ of $G$ in the sense of Dynkin [2]. If a Lie group $G$ has $n$ simple factors, then $\pi_{3}(G)$ is isomorphic to a free abelian group of rank $n$, that is, $\pi_{3}(G)=\oplus^{n} \mathbf{Z}$. Therefore in the above cases we can regard $j_{*}: \pi_{3}(H) \rightarrow \pi_{3}(G)$ as multiplication by an integer $n$ or a pair of integers $(n, m)$ associated to the inclusion $j: H \rightarrow G$. The assertion of [9, Lemma 4] guarantees that the index of the subgroup $H$ of $G$ can be interpreted as the above integer or pair of integers determined by the inclusion $j$.

In order to prove Theorem 1.1 , we need to consider the injectivity of the $D$ map $\mathscr{D}_{X}: H^{4}(X ; \mathbf{Z}) \rightarrow H^{3}(L X ; \mathbf{Z})$. To this end, we study the algebra structure of $H^{*}(L X ; \mathbf{Z} / p)$ and the injectivity of the $\bmod p D$-map $\mathscr{D}_{X, p}=\int_{S^{1}} \propto e v^{*}$ : $H^{4}(X ; \mathbf{Z} / p) \rightarrow H^{3}(L X ; \mathbf{Z} / p)$ for any prime $p$. The behavior of $\mathscr{D}_{X . p}$ in $H^{4}(X ; \mathbf{Z} / p)$ is determined by Theorem 1.3 and Theorem 1.4. We note that the $D$-map $\mathscr{D}_{X}$ is a derivation (see [5, Section 3]). More precisely, $\mathscr{D}_{X}(x y)=\mathscr{D}_{X}(x) y+(-1)^{\operatorname{deg} x} x \mathscr{D}_{X}(y)$. Here $H^{*}(L X ; \mathbf{Z})$ is regarded as a two sided $H^{*}(X ; \mathbf{Z})$-module via the homomorphism induced from the evaluation map $\pi: L X \rightarrow X$ at zero.

NOTATION. Let $\Lambda$ be a graded algebra and $S$ a subset of $\Lambda$. Then the ideal of $\Lambda$ generated by elements of $S$ will be denoted by $(S)_{\Lambda}$. For any graded vector space $V=\oplus_{i \geq 0} V^{i}, V \leq n$ means $\oplus_{0 \leq i \leq n} V^{i}$. We denote the commutative algebra with the 2-simple system of generators $\left\{z_{j}\right\}_{j=1 \ldots . . n}$ by $\Delta\left(z_{1}, \ldots z_{n}\right)$. Let $T$ be a subset of a vector space $W$ over a field $\mathbf{k}$. We denote the subspace of $W$ generated by elements of $T$ by $\mathbf{k}\{T\}$.

THEOREM 1.3. Suppose that $X$ is a simply connected space and that there exists a morphism of algebras

$$
\phi: B=\Lambda\left(y_{1}, \ldots, y_{i}\right) \otimes \mathbf{Z} / p\left[x_{1}, \ldots, x_{n}\right] /\left(\rho_{1}, \ldots, \rho_{m}\right) \rightarrow H^{*}(X ; \mathbf{Z} / p),
$$

which is an isomorphism below degree 4 , where $\rho_{1}, \ldots, \rho_{n}$ are decomposable elements with degree $4, \operatorname{deg} x_{j}=2$ or $4, \operatorname{deg} y_{i}=3$ and $l=0, \operatorname{deg} x_{j}=2,3$ or 4 if $p=2$. We regard $H^{*}(L X ; \mathbf{Z} / p)$ as a $B$-module via the composition map $\pi^{*} \phi$. Then there exists a morphism of algebras and of $B$-modules

$\psi: \Lambda\left(y_{1}, \ldots, y_{l}\right) \otimes \Gamma\left[\bar{y}_{1}, \ldots, \bar{y}_{l}\right] \otimes\left\{A_{p} /\left(d\left(\omega_{1}\right), \ldots, d\left(\omega_{m}\right)\right)_{A_{p}}\right\} \rightarrow H^{*}(L X ; \mathbf{Z} / p)$ 
which is a monomorphism below degree 3 , where $d\left(\omega_{i}\right)=\sum_{j=1}^{n}\left(\partial \rho_{i} / \partial x_{j}\right) \bar{x}_{j}, \operatorname{deg} \bar{x}_{j}=$ $\operatorname{deg} x_{j}-1, \operatorname{deg} \bar{y}_{i}=2, \operatorname{deg} \omega_{i}=\operatorname{deg} \rho_{i}-2$ and $A_{p}=\mathbf{k}_{p}\left[x_{1}, \ldots, x_{n}\right] /\left(\rho_{1}, \ldots, \rho_{m}\right) \otimes$ $\Lambda\left(\bar{x}_{1}, \ldots, \bar{x}_{n}\right)$ if $p \neq 2$, and $A_{2}=\mathbf{k}_{2}\left[x_{1}, \ldots, x_{n}\right] /\left(\rho_{1}, \ldots, \rho_{m}\right) \otimes \Delta\left(\bar{x}_{1}, \ldots, \bar{x}_{n}\right)$ if $p=2$, where $\Delta\left(\bar{x}_{1}, \ldots, \bar{x}_{n}\right)$ is the commutative algebra with the 2-simple system of generators $\left\{\bar{x}_{j}\right\}_{j=1 \ldots . . . n}$.

We identify the elements $y_{j}$ and $x_{i}$ with $\phi\left(y_{j}\right)$ and $\phi\left(x_{i}\right)$, respectively. Let $\sigma^{*}$ : $H^{*}(X ; \mathbf{Z} / p) \rightarrow H^{*-1}(\Omega X ; \mathbf{Z} / p)$ be the cohomology suspension and $i: \Omega X \rightarrow L X$ the inclusion map.

THEOREM 1.4. One can choose the elements $\bar{y}_{i}$ and $\bar{x}_{j}$ in Theorem 1.3 so that $i^{*}\left(\bar{y}_{i}\right)=\sigma^{*}\left(y_{i}\right), i^{*}\left(\bar{x}_{j}\right)=\sigma^{*}\left(x_{j}\right)$ and

$$
\mathscr{D}_{X .\left.p\right|_{H^{4}(X: Z / / p)}}=\sum_{j=1}^{n} \frac{\partial}{\partial x_{j}} \bar{x}_{j} .
$$

Notice that, for any simply connected space $X$, one can construct an algebra and a morphism of algebras $\phi$ satisfying the condition of Proposition 1.3 by using indecomposable elements $x_{j}$ and $y_{i}$ in $H^{*}(X ; \mathrm{Z} / p)$.

This paper is organized as follows. In section 2 , Theorem 1.2 is proved by applying Theorem 1.1. Our main tool to prove Theorem 1.3 is the Eilenberg-Moore spectral sequence converging to $H^{*}(L X ; \mathbf{Z} / p)$ whose $E_{2}$-term is isomorphic to the bigraded Hochschild homology of $H^{*}(X ; \mathbf{Z} / p)$. We will determine the indecomposable elements in $H^{*}(X ; \mathbf{Z} / p)$ with degree below 3 and relations between the elements via the $E_{2}$-term of the spectral sequence. To this end, Section 3 is devoted to studying the Hochschild homology below degree 3 of a commutative algebra. In section 4 , Theorem 1.3 and Theorem 1.4 are proved. Moreover we study the structure of the kernel of the $\bmod p D$-map $\mathscr{D}_{X . p}: H^{4}(X ; \mathbf{Z} / p) \rightarrow H^{3}(L X ; \mathbf{Z} / p)$ as a vector space. Finally, by using Theorem 1.3 and Theorem 1.4, we prove Theorem 1.1.

The authors are grateful to Professor Akira Kono for helpful conversations on the proof of Theorem 1.4 .

\section{Proof of Theorem 1.2}

Let $X$ and $Y$ be simply connected spaces satisfying the condition (1.1). By the Universal Coefficient Theorem, we see that $H^{2}(X ; \mathbf{Z})$ and $H^{2}(Y ; \mathbf{Z})$ are torsion free. Hence it follows from the Künneth Theorem that $H^{4}(X \times Y ; Z)$ is isomorphic to $\oplus_{i+j=4} H^{i}(X ; \mathbf{Z}) \otimes H^{j}(Y ; \mathbf{Z})$. As a consequence, the product space $X \times Y$ also satisfies the condition (1.1). It is clear that if $X$ and $Y$ satisfy the condition (1.2) then $X \times Y$ also satisfy the condition (1.2). Let $M$ be a simply connected 4-dimensional 
manifold. Since $H^{4}(M ; \mathbf{Z})$ is isomorphic to $\mathbf{Z}$, it follows that $M$ satisfies the condition (1.1). Thus, in order to prove Theorem 1.2, it suffices to show that any compact, simply connected homogeneous space of rank one satisfies the conditions (1.1) and (1.2).

PROPOSITION 2.1. Any compact, simply connected homogeneous space of rank one satisfies the conditions (1.1) and (1.2).

PROOF. It is clear that spheres and projective spaces satisfy (1.1) and (1.2). We will show that the nine homogeneous spaces listed in Section 1 satisfy (1.1) and (1.2).

From the computation of the cohomology of the homogeneous spaces $M$ by McCleary and Ziller [7, Theorem 1], one can conclude that $H^{4}(M ; Z)$ is torsion free for the cases (1), (3), (5), (6) and (8).

We consider the case (9). Let $\pi: \operatorname{Spin}(n) \rightarrow S O(n)$ be the universal covering. By the Hurewicz theorem, $(j \pi)_{*}: H_{3}(\operatorname{Spin}(3) ; \mathbf{Z}) \rightarrow H_{3}\left(G_{2} ; \mathbf{Z}\right)$ is multiplication by 28. In order to prove that $j_{*}: H_{3}(S O(3) ; Z) \rightarrow H_{3}\left(G_{2} ; Z\right)$ is multiplication by 14 , we will show that $\pi_{*}: H_{3}(\operatorname{Spin}(3) ; \mathbf{Z}) \rightarrow H_{3}(S O(3) ; Z)$ is multiplication by 2 . Let us consider the homology Leray-Serre spectral sequence $\left\{\hat{E}_{*, *}^{r}, \hat{d}^{r}\right\}$ of the universal $S O(n)$-bundle. Since $\hat{E}_{2,1}^{2}=H_{2}\left(B S O(3) ; H_{1}(S O(3) ; \mathbf{Z})\right)=H_{2}(B S O(3) ; \mathbf{Z} / 2)=$ $\mathbf{Z} / 2$ and $\hat{E}_{4.0}^{2}=H_{4}(B S O(3) ; Z)=Z$, it follows that $\hat{E}_{4.0}^{3}=2 Z$. Therefore we can deduce $\hat{d}^{3}: \hat{E}_{4.0}^{3} \rightarrow \hat{E}_{0.3}^{3}$ is multiplication by $1 / 2$. Note that $\hat{E}_{0.3}^{3}=H_{3}(S O(3) ; \mathbf{Z})=$ Z. The index of the map $B(\pi)^{*}: H^{4}(B S O(3) ; \mathbf{Z})=\mathbf{Z} \rightarrow H^{4}(B S p i n(3) ; \mathbf{Z})=\mathbf{Z}$ is 4. From the Universal Coefficient Theorem, it follows that the index of the map $B(\pi)_{*}: H_{4}(B \operatorname{Spin}(3) ; \mathbf{Z}) \rightarrow H_{4}(B S O(3) ; Z)$ is 4 also. Thus the naturality of the differential in the spectral sequence enables us to conclude that the index of $\pi_{*}$ is 2. Hence we see $j_{*}: H_{3}(S O(3) ; \mathbf{Z}) \rightarrow H_{3}\left(G_{2} ; \mathbf{Z}\right)$ is multiplication by 14 . To prove that the homogeneous space $M=\left(G_{2}, S O(3), 28\right)$ satisfies the condition (1.1), we consider the homology Leray-Serre spectral sequence $\left\{E_{*, *}^{r}, d^{r}\right\}$ of the fibration $S O(3) \rightarrow G_{2} \rightarrow G_{2} / S O(3)=M$. Let $\left\{F_{p} H_{*}\right\}_{p \geq 0}$ be the filtration of $H_{*}\left(G_{2} ; \mathbf{Z}\right)$ which comes from the spectral sequence $\left\{E_{*, *}^{r}, d^{r}\right\}$. Notice that $j_{*}$ coincides with the boundary homomorphism

$$
H_{3}(S O(3) ; \mathbf{Z})=E_{0.3}^{2} \rightarrow E_{0.3}^{\infty} \cong E_{0.3}^{0 .}=F_{0} H_{3} \subset F_{1} H_{3} \subset F_{2} H_{3} \subset F_{3} H_{3}=H_{3}\left(G_{2} ; \mathbf{Z}\right) \text {. }
$$

Since $E_{0.2}^{2}=H_{2}(S O(3) ; \mathbf{Z})=0$ and $E_{1,1}^{2}=0$, we obtain $H_{3}(M ; Z)=E_{3,0}^{2}=E_{3.0}^{\infty} \cong$ $F_{3} H_{3} / F_{2} H_{3}$. From [7, Theorem 1 (9)], it follows that $E_{2,1}^{2}=\mathrm{Z} / 2$ and $H_{4}(M ; Z)$ does not have a 2-torsion part and a free part. Therefore we see $\mathbf{Z} / 2=E_{2,1}^{2} \cong$ $E_{2.1}^{\infty} \cong F_{2} H_{3} / F_{1} H_{3}$. The fact that the index of $j$ is non-zero and $E_{0.3}^{0}$ is a subgroup of $H_{3}\left(G_{2} ; \mathbf{Z}\right)=\mathbf{Z}$ allows us to deduce $E_{0.3}^{2} \cong E_{0.3}^{\infty}$. Since $M$ is simply connected, $E_{1.2}^{2}=0$. Hence $j_{*}$ coincides with the inclusion $\mathbf{Z}=H_{3}(S O(3) ; \mathbf{Z})=E_{0.3}^{2} \cong E_{0.3}^{\infty} \cong$ $E_{0.3}^{0}=F_{0} H_{3}=F_{1} H_{3} \subset F_{2} H_{3} \subset F_{3} H_{3}=H_{3}\left(G_{2} ; Z\right)$. The above argument yields 
that the inclusion $\mathbf{Z}=F_{1} H_{3} \rightarrow F_{2} H_{3}=\mathbf{Z}$ is multiplication by 2 . Since the index of $j_{*}$ is 14 , we have the inclusion $\mathbf{Z}=F_{2} H_{3} \rightarrow F_{3} H_{3}=\mathbf{Z}$ is multiplication by 7 . It turns out that $H_{3}(M ; Z)=\mathbf{Z} / 7$. By using the Universal Coefficient Theorem, we see the manifold $M=G_{2} / S O(3)$ satisfies the condition (1.1).

The same argument works in cases (2), (4) and (7). From [7, Theorem 1], we obtain that the only case where $H^{4}(M ; \mathbf{Z})$ has 2-torsion is $(4):(G, H, i)=$ $(S p(2), S U(2), 10)$. It is clear that the manifold satisfies the condition (1.2) since the manifold $S p(2) / S U(2)$ is 2-connected.

REMARK 2.2. For the manifolds $M$ in the cases (1), (3), (5), (6) and (8), $H^{4}(M ; \mathbf{Z})$ is torsion free. Therefore, by virtue of Theorem A, we can deduce that the $D$-maps of these manifolds are good. Since the manifolds in the case (2), (4) and (7) are 2-connected, it follows from [8, Theorem 3.1] that the manifolds have a good $D$-map. However, we cannot conclude that the manifold in the case (9) and product spaces of compact, simply connected homogeneous spaces of rank one have a good $D$-map by applying Theorem A or [8, Theorem 3.1].

\section{The Hochschild homology below degree 3}

The purpose of this section is to prepare the proof of Theorem 1.3. In order to consider the algebra structure of $H^{*}(L X ; \mathbf{Z} / p)$, we use the Eilenberg-Moore spectral sequence converging to $H^{*}(L X ; Z / p)$ whose $E_{2}$-term is isomorphic to the Hochschild homology of $H^{*}(X ; \mathbf{Z} / p)$. Before we begin calculating this spectral sequence, we give an available complex to determine the algebra structure of the Hochschild homology of a certain commutative algebra. For details of the Hochschild homology of commutative differential graded algebras, see [1]. For the rest of this paper, a commutative algebra $A$ will mean a positive graded commutative algebra over $\mathbf{Z} / p$ such that $A^{0}=\mathbf{Z} / p$ and $A^{1}=0$. Let $\Lambda$ be a commutative algebra $\Lambda\left(y_{1}, \ldots, y_{l}\right) \otimes \mathbf{Z} / p\left[x_{1}, \ldots, x_{n}\right] /\left(\rho_{1}, \ldots, \rho_{m}\right)$, where $\rho_{i}$ is decomposable for any $i$. We will suppose that $2 \leq \operatorname{deg} x_{1} \leq \cdots \leq \operatorname{deg} x_{n}, 3 \leq \operatorname{deg} y_{1} \leq \cdots \leq \operatorname{deg} y_{l}$, $\operatorname{deg} \rho_{1} \leq \cdots \leq \operatorname{deg} \rho_{m}$ and $l=0$ if $p=2$. If $\rho_{1}, \ldots, \rho_{m}$ is a regular sequence, the Koszul-Tate complex (see [11], [3, Proposition 1.1]) of $\Lambda$ is a complex for computing the Hochschild homology $H H_{*}(\Lambda)$. In the general case, we can also obtain a complex for computing $H H_{*}(\Lambda)$ by extending the Koszul-Tate complex.

PROPOSITION 3.1. The Hochschild homology of $\Lambda$ is calculable as the homology of the following complex $(\mathscr{E}, d)$ :

$$
\mathscr{E}:=\Lambda \otimes \Gamma\left[\bar{y}_{1}, \ldots, \bar{y}_{l}\right] \otimes \Lambda\left(\bar{x}_{1}, \ldots, \bar{x}_{n}\right) \otimes \Gamma\left[\omega_{1}, \ldots, \omega_{m}\right] \otimes \mathscr{C},
$$


$d\left(\omega_{i}\right)=\sum_{j=1}^{n}\left(\partial \rho_{i} / \partial x_{j}\right) \bar{x}_{j}, d(\lambda)=d\left(\bar{y}_{i}\right)=d\left(\bar{x}_{j}\right)=0$ for $\lambda \in \Lambda, i=1, \ldots, l$, $j=1, \ldots, n$ and bideg $\lambda=(0, \operatorname{deg} \lambda)$ for $\lambda \in \Lambda, \operatorname{bideg} \bar{x}_{j}=\left(-1, \operatorname{deg} x_{j}\right), \operatorname{bideg} \bar{y}_{i}=$ $\left(-1, \operatorname{deg} y_{i}\right)$, bideg $\omega_{i}=\left(-2, \operatorname{deg} \rho_{i}\right)$. Here $\mathscr{C}$ is a suitable differential graded algebra which is a tensor product of an exterior algebra and a divided power algebra. Moreover, the differential d satisfies

$$
\begin{aligned}
\{d \mathscr{E} \cap \Lambda \otimes & \left.\Gamma\left[\bar{y}_{1}, \ldots, \bar{y}_{l}\right] \otimes \Lambda\left(\bar{x}_{1}, \ldots, \bar{x}_{n}\right)\right\}^{\leq 3} \\
= & \mid d\left(\Lambda \otimes \Gamma\left[\bar{y}_{1}, \ldots, \bar{y}_{l}\right] \otimes \Lambda\left(\bar{x}_{1}, \ldots, \bar{x}_{n}\right) \otimes \Gamma\left[\omega_{1}, \ldots, \omega_{m}\right]\right) \\
& \left.\quad \sim \wedge \& \Gamma\left[\bar{y}_{1}, \ldots, \bar{y}_{l}\right] \otimes \Lambda\left(\bar{x}_{1}, \ldots, \bar{x}_{n}\right)\right\}^{\leq 3} .
\end{aligned}
$$

PROOF. Let $A$ and $B$ denote the commutative algebra $\Lambda\left(y_{1}, \ldots, y_{l}\right)$ and $\mathbf{Z} / p\left[x_{1}, \ldots, x_{n}\right] /\left(\rho \ldots \rho_{m}\right)$ respectively. If $\rho_{1}, \ldots, \rho_{m}$ is a regular sequence, then there exists the follom in moper projective resolution $\mathscr{F} \stackrel{\mu}{\longrightarrow} B \longrightarrow 0$ of $B$ as a left $B \otimes B$-module ([1]| [3. Proposition 1.1]):

$$
\bar{F}=B \because B \otimes \Lambda\left(\bar{x}_{1}, \ldots, \bar{x}_{n}\right) \otimes \Gamma\left[\omega_{1}, \ldots, \omega_{m}\right],
$$

$\mu: B \otimes B \rightarrow B$ w the muluplication of $B, d\left(\bar{x}_{j}\right)=x_{j} \otimes 1-1 \otimes x_{j}$ and $d\left(\omega_{i}\right)=$ $\sum_{j=1}^{n} \zeta_{i j} \bar{x}_{j}$. where: 1 an element in $B \otimes B$ satisfying $\rho_{i} \otimes 1-1 \otimes \rho_{i}=\sum_{j=1}^{n} \zeta_{i j}\left(x_{j} \otimes\right.$ $1-1 \otimes x$, ) and $\mu 1:=\omega_{1} \quad \dot{\partial} x_{;}$.

In particular. we csn ithune the element $\sum_{k=1}^{n} \mu_{j k}^{(i)} x_{k} \otimes 1+\sum_{k=1}^{n} 1 \otimes \mu_{k j}^{(i)} x_{k}$ as the element $\zeta_{1}$, mentumat atuine it $\rho=\sum_{j=1}^{n} \sum_{k=1}^{n} \mu_{j k}^{(i)} x_{k} x_{j}$.

Let us consider the pence sl cave where $\rho_{1}, \ldots, \rho_{m}$ are decomposable elements. By modifying the methind …. nuvtruct a minimal model of a differential graded algebra, we obtain the required Jift erentisl graded algebra $\mathscr{E}$. The argument of [11, Lemma 3.3] enables us to deduce that $H \cdot i_{1}=0$. When $i+j=1$, every element in $\mathscr{F}^{i, j}$ can be written by a linesi cumbination of elements $\bar{x}_{1}, \ldots, \bar{x}_{n}$. Therefore, $H^{i, j}(\mathscr{F})=0$ for $i+j=1$. Suppose that $i+j=2$ and $H^{i, j}(\mathscr{F}) \neq 0$. Then, from the definition of the differential $d$. ue obrain $(i, j)=(-2,4)$. If the element $u=\sum_{i<j} a_{i j} \bar{x}_{i} \bar{x}_{j}$ is in $\operatorname{Ker} d^{-2.4}$, then $0=d u=\sum \ldots, a_{i j}\left(x_{1} \otimes 1-1 \otimes x_{i}\right) \bar{x}_{j}-\sum_{i<j} a_{i j}\left(x_{j} \otimes 1-1 \otimes x_{j}\right) \bar{x}_{i}$. Thus we see that $a_{1 n}\left(x_{1} \otimes 1-1 \otimes x_{1}\right)+\cdots+a_{n-1}\left(x_{n-1} \otimes 1-1 \otimes x_{n-1}\right)=0$ and hence $a_{i n}=0$ for any $i<n$. Inductively, we have $a_{i j}=0$ for any $i$ and $j$. From this fact, we can conclude that each element of a basis $\left\{z_{1}, \ldots, z_{s}\right\}$ for $H^{-2.4}(\mathscr{F})$ represents an element $\sum a_{i j} \bar{x}_{i} \bar{x}_{j}+\sum b_{k} w_{k}$, where $b_{k}$ is nonzero for some $k$. The element $z_{\alpha}$ and its representative element will be denoted by the same notation. We define the differential graded algebra $\left(\mathscr{F}_{1}, d\right)$ by $\mathscr{F}_{1}=\mathscr{F} \otimes \Lambda\left(\bar{z}_{\alpha}\right)$ and $d\left(\bar{z}_{\alpha}\right)=z_{\alpha}$, where bideg $z_{\alpha}=$ $(-3,4)$. Clearly, $H^{i, j}\left(\mathscr{F}_{1}\right)=0$ for $i+j=2$. From the form of a representative element of $z_{\alpha}$, it follows that $d\left(\Lambda\left(\bar{z}_{\alpha}\right)\right) \cap B \otimes B \otimes \Lambda\left(\bar{x}_{1}, \ldots, \bar{x}_{n}\right)=0$. Consider the case where $i+j=3$. It is easy to verify that $\operatorname{Ker} d^{-2.5} \cap \mathbf{Z} / p\left\{\bar{x}_{i} \bar{x}_{j} ; 1 \leq i, j \leq n\right\}=0$ in the only case where $p=2$ and that $\operatorname{Ker} d^{-3.6} \cap \mathbf{Z} / p\left\{\bar{x}_{i} \bar{x}_{j} \bar{x}_{k} ; 1 \leq i, j, k \leq n\right\}=0$. We define the elements $\bar{v}_{\beta}$ with total degree 3 corresponding to representative elements 
$v_{\beta}$ of a basis of $H^{i, j}\left(\mathscr{F}_{1}\right)$ for $(i, j)=(-2,5)$ and $(-3,6)$. Put $\mathscr{F}_{2}=\mathscr{F}_{1} \otimes \Gamma\left[\bar{v}_{\beta}\right]$ and extend the differential $d$ by demanding that $d\left(\bar{v}_{\beta}\right)=v_{\beta}$. The elements of $\operatorname{Ker} d \cap \mathscr{F}_{1}^{-1,4}$ are characterized as follows:

LEMMA 3.2. Let $u$ be an element of $\operatorname{Ker} d \cap \mathscr{F}_{1}^{-1,4}$. Then $u$ can be written as $\sum_{j=1}^{n}\left(\sum_{k=1}^{n} \lambda_{j k} x_{k} \otimes 1+\sum_{k=1}^{n} 1 \otimes \lambda_{k j} x_{k}\right) \bar{x}_{j}$ with coefficients $\lambda_{j k}$ satisfying $\lambda_{j j}=$ $\alpha^{(1)} \mu_{j j}+\cdots+\alpha^{(m)} \mu_{j j}$ and $\left(\lambda_{j k}+\lambda_{k j}\right)=\alpha^{(1)}\left(\mu_{j k}^{(1)}+\mu_{k j}^{(1)}\right)+\cdots+\alpha^{(m)}\left(\mu_{j k}^{(m)}+\mu_{k j}^{(m)}\right)$ for some $\alpha^{(i)}\left(\right.$ for the notation $\mu_{j k}^{(l)}$ see the definition of the above resolution $\left.\mathscr{F}\right)$. Therefore, the element $1 \otimes_{\Lambda \otimes \Lambda} u$ in $\Lambda \otimes_{\Lambda \otimes \Lambda} \mathscr{F}_{1}$ belongs to $\mathrm{Z} / p\left\{d\left(\omega_{1}\right), \ldots, d\left(\omega_{m}\right)\right\}$.

Let $\left\{u_{\alpha}\right\}$ be a basis for $H^{-1.4}\left(\mathscr{F}_{1}\right)$. We extend the complex $\mathscr{F}_{2}$ to $\mathscr{F}_{3}=\mathscr{F}_{2} \otimes$ $\Gamma\left[\bar{u}_{\alpha}\right]=\mathscr{F}_{1} \otimes \Gamma\left[\bar{v}_{\beta}\right] \otimes \Gamma\left[\bar{u}_{\alpha}\right]$ with the differential defined by $d\left(\bar{u}_{\alpha}\right)=u_{\alpha}$. From this construction, we see that $H^{i, j}\left(\mathscr{F}_{2}\right)=0$ for $i+j=3$ and $d\left(\Gamma\left[\bar{v}_{\beta}\right]\right) \cap B \otimes B \otimes$ $\Lambda\left(\bar{x}_{1}, \ldots, \bar{x}_{n}\right)=0$. By continuing the same process above total degree 4 , we can get a proper projective resolution $\widetilde{\mathscr{E}}_{B}$ of $B$ as a $B \otimes B$-module : $\widetilde{\mathscr{E}}_{B}=\mathscr{F} \otimes \mathscr{C}$. By virtue of [11, Lemma 3.2], we conclude that the differential graded algebra $\left(\widetilde{\mathscr{E}}_{A}, d\right)$, defined by $\widetilde{\mathscr{E}}_{A}=A \otimes A \otimes \Gamma\left[\bar{y}_{1}, \ldots \bar{y}_{l}\right]$ and $d\left(\bar{y}_{i}\right)=y_{i} \otimes 1-1 \otimes y_{i}$, is a proper projective resolution of $A$ as an $A \otimes A$-module. Therefore, the differential graded algebra $\widetilde{E}=\widetilde{\mathscr{E}}_{A} \otimes \widetilde{\mathscr{E}}_{B}$ is a proper projective resolution of $\Lambda$ as a $\Lambda \otimes \Lambda$-module. Thus the Hochschild homology $H H_{*, *}(\Lambda)=\operatorname{Tor}_{\Lambda \otimes \Lambda}^{* * *}(\Lambda, \Lambda)$ is obtained as the homology of the complex $(\mathscr{E}, d)=\left(\Lambda \otimes_{\Lambda \otimes \Lambda} \tilde{\mathscr{E}}, 1 \otimes d\right)$. From Lemma 3.2, it follows that $d\left(\bar{u}_{\alpha}\right)=1 \otimes_{\Lambda \otimes \Lambda} d\left(\bar{u}_{\alpha}\right)=1 \otimes_{\Lambda \otimes \Lambda} u_{\alpha}$ is in $\mathbf{Z} / p\left\{d\left(\omega_{1}\right), \ldots, d\left(\omega_{m}\right)\right\}$. This fact and the definitions of $d\left(\bar{z}_{\alpha}\right)$ and $d\left(\bar{v}_{\beta}\right)$ allow us to deduce that

$$
\begin{aligned}
\{d \mathscr{E} & \left.\cap \Lambda \otimes \Gamma\left[\bar{y}_{1}, \ldots, \bar{y}_{l}\right] \otimes \Lambda\left(\bar{x}_{1}, \ldots, \bar{x}_{n}\right)\right\}^{\leq 3} \\
& =\left\{d\left(\Lambda \otimes_{\Lambda \otimes \Lambda}\left(\widetilde{\mathscr{E}}_{A} \otimes \mathscr{F}\right)\right) \cap \Lambda \otimes \Gamma\left[\bar{y}_{1}, \ldots, \bar{y}_{l}\right] \otimes \Lambda\left(\bar{x}_{1}, \ldots, \bar{x}_{n}\right)\right\}^{\leq 3} .
\end{aligned}
$$

This completes the proof of Proposition 3.1.

PROOF OF LEMMA 3.2. For any element $u \in \operatorname{Ker} d \cap \mathscr{F}_{1}^{-1.4}$, we can write $u=$ $\sum_{j} \xi_{j} \bar{x}_{j}$, where $\xi_{j}=\sum_{k=1}^{n} \lambda_{j k} x_{k} \otimes 1+\sum_{k=1}^{n} 1 \otimes \lambda_{j k}^{\prime} x_{k}$. Since $d(u)=0$ in $\mathscr{F}_{1}^{0.4}=$ $\mathscr{F}^{0.4}$, it follows that the element

$$
\begin{aligned}
d u=\sum_{j=1}^{n} & \sum_{k=1}^{n} \lambda_{j k} x_{k} x_{j} \otimes 1-\sum_{j=1}^{n} \sum_{k=1}^{n} \lambda_{j k} x_{k} \otimes x_{j}+\sum_{j=1}^{n} \sum_{k=1}^{n} \lambda_{j k}^{\prime} x_{j} \otimes x_{k} \\
& -\sum_{j=1}^{n} \sum_{k=1}^{n} 1 \otimes \lambda_{j k}^{\prime} x_{k} x_{j}
\end{aligned}
$$

belongs to the ideal $\left(\rho_{i} \otimes 1,1 \otimes \rho_{i} ; 1 \leq i \leq m\right)$ in $\mathbf{Z} / p\left[x_{1}, \ldots, x_{n}\right] \otimes \mathbf{Z} / p\left[x_{1}, \ldots, x_{n}\right]$. This fact enables us to conclude that $\sum_{j=1}^{n} \sum_{k=1}^{n} \lambda_{j k} x_{k} \otimes x_{j}=\sum_{j=1}^{n} \sum_{k=1}^{n} \lambda_{j k}^{\prime} x_{j} \otimes x_{k}$ and so $\lambda_{j k}=\lambda_{k j}^{\prime}$. Moreover we see $\sum_{j=1}^{n} \sum_{k=1}^{n} \lambda_{j k} x_{k} x_{j}=\alpha^{(1)} \rho_{1}+\cdots+\alpha^{(m)} \rho_{m}=$ 
$\alpha^{(1)}\left(\sum_{j=1}^{n} \sum_{k=1}^{n} \mu_{j k}^{(1)} x_{k} x_{j}\right)+\cdots+\alpha^{(m)}\left(\sum_{j=1}^{n} \sum_{k=1}^{n} \mu_{j k}^{(m)} x_{k} x_{j}\right)$ in $\mathbf{Z} / p\left[x_{1}, \ldots, x_{n}\right]$. Thus we have

$$
\begin{aligned}
\sum_{j=1}^{n} \lambda_{j j} x_{j}^{2}+\sum_{j<k}\left(\lambda_{j k}+\lambda_{k j}\right) x_{k} x_{j}= & \alpha^{(1)}\left(\sum_{j=1}^{n} \mu_{j j}^{(1)} x_{j}^{2}+\sum_{j<k}\left(\mu_{j k}^{(1)}+\mu_{k j}^{(1)}\right) x_{k} x_{j}\right)+\cdots \\
& +\alpha^{(m)}\left(\sum_{j=1}^{n} \mu_{j j}^{(m)} x_{j}^{2}+\sum_{j<k}\left(\mu_{j k}^{(m)}+\mu_{k j}^{(m)}\right) x_{k} x_{j}\right)
\end{aligned}
$$

in $\mathbf{Z} / p\left[x_{1}, \ldots, x_{n}\right]$. Therefore, the required relations for $\lambda_{j k}$ are obtained. Let $\mu$ be the multiplication of $B$. Since $\mu\left(\zeta_{i j}\right)=\sum_{k=1}^{n}\left(\mu_{j k}^{(i)}+\mu_{k j}^{(i)}\right) x_{k}$, it follows that

$$
\begin{aligned}
1 \otimes_{\Lambda \otimes \Lambda} u & =\sum_{j=1}^{n}\left(\sum_{k=1}^{n}\left(\lambda_{j k}+\lambda_{k j}\right) x_{k}\right) \bar{x}_{j} \\
& =\sum_{j=1}^{n} \alpha^{(1)}\left(\sum_{k=1}^{n}\left(\mu_{j k}^{(1)}+\mu_{k j}^{(1)}\right) x_{k}\right) \bar{x}_{j}+\cdots+\sum_{j=1}^{n} \alpha^{(m)}\left(\sum_{k=1}^{n}\left(\mu_{j k}^{(m)}+\mu_{k j}^{(m)}\right) x_{k}\right) \bar{x}_{j} \\
& =\sum_{j=1}^{n} \alpha^{(1)} \mu\left(\zeta_{1 j}\right) \bar{x}_{j}+\cdots+\sum_{j=1}^{n} \alpha^{(m)} \mu\left(\zeta_{m j}\right) \bar{x}_{j} \\
& =\alpha^{(1)} d\left(\omega_{1}\right)+\cdots+\alpha^{(m)} d\left(\omega_{m}\right) .
\end{aligned}
$$

Thus we have Lemma 3.2.

Applying Proposition 3.1, we can partially know the algebra structure of the Hochschild homology of the graded algebra $\Lambda$.

PROPOSITION 3.3. Let $\Lambda$ be the graded algebra $\Lambda\left(y_{1}, \ldots, y_{l}\right) \otimes \mathbf{Z} / p\left[x_{1}, \ldots, x_{n}\right] /$ $\left(\rho_{1}, \ldots, \rho_{m}\right)$. Then there exists a morphism of algebras

$$
\phi: \Lambda\left(y_{1}, \ldots, y_{l}\right) \otimes \Gamma\left[\bar{y}_{1}, \ldots, \bar{y}_{l}\right] \otimes\left\{A /\left(d \omega_{1}, \ldots, d \omega_{m}\right)_{A}\right\} \rightarrow H H_{*, *}(\Lambda)
$$

which is a monomorphism below total degree 3 , where $A=\mathbf{Z} / p\left[x_{1}, \ldots x_{n}\right] /$ $\left(\rho_{1}, \ldots, \rho_{m}\right) \otimes \Lambda\left(\bar{x}_{1}, \ldots, \bar{x}_{n}\right)$. 
PROOF. Let us consider the following commutative diagram:

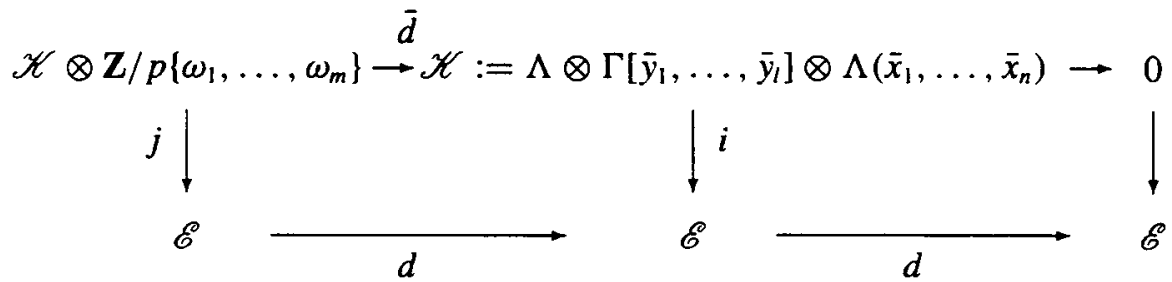

where $i$ and $j$ are the inclusion maps and $\bar{d}$ is the restriction of $d$ to $\mathscr{K} \otimes \mathbf{Z} / p\left\{\omega_{1}, \ldots\right.$, $\left.\omega_{m}\right\}$. Suppose that $H(i)[z]=0$ and the total degree of $[z]$ is below 3. Then $i(z)$ belongs to $\{d(\mathscr{E}) \cap \mathscr{K}\}^{\leq 3}$. From Proposition 3.1, we can see that $i(z)$ is in the vector space $\{\operatorname{Im} d \circ j\}^{\leq 3}$. Therefore $z$ is an element in $\operatorname{Im} \bar{d}$. The map $\phi=H(i)$ is the demanded homomorphism.

\section{Kernel of the $D$-map $\mathscr{D}_{X, p}$}

Let $X$ be a simply-connected space. In order to study the structure of the kernel of $\mathscr{D}_{X . p}=\int_{S^{1}} \circ e v^{*}: H^{4}(X ; \mathbf{Z} / p) \rightarrow H^{3}(L X ; \mathbf{Z} / p)$, it is important to consider the ring structure of $H^{*}(L X ; \mathbf{Z} / p)$, in particular, to clarify indecomposable elements with degree below 3 in $H^{*}(L X ; \mathbf{Z} / p)$ and relations between their elements in $H^{3}(L X ; \mathbf{Z} / p)$. To this end, we use the Eilenberg-Moore spectral sequence converging to $H^{*}(L X ; \mathbf{Z} / p)([11],[3])$ whose $E_{2}$-term is the Hochschild homology of $H^{*}(X ; \mathbf{Z} / p)$ :

$$
E_{2}^{* * *} \cong \operatorname{Tor}_{H^{*}(X: \mathbf{Z} / p) \otimes H^{*}(X: \mathbf{Z} / p)}^{* *}\left(H^{*}(X ; \mathbf{Z} / p), H^{*}(X ; \mathbf{Z} / p)\right)=H H\left(H^{*}(X ; \mathbf{Z} / p)\right) .
$$

Proposition 3.3 plays an important role in explaining the algebra structure of $H^{*}(L X$; $\mathrm{Z} / \mathrm{p}$ ). Before we prove Theorem 1.3, we prepare a lemma.

LEMMA 4.1. Let $C_{1}$ and $C_{2}$ be commutative algebras. Suppose that there exists a morphism of algebras $\theta: C_{1} \rightarrow C_{2}$ which is an isomorphism below degree $s$. Then $\operatorname{Tor}_{\theta \otimes \theta}(\theta, \theta): \operatorname{Tor}_{C_{1} \otimes C_{1}}^{i . j}\left(C_{1}, C_{1}\right) \rightarrow \operatorname{Tor}_{C_{2} \otimes C_{2}}^{i . j}\left(C_{2}, C_{2}\right)$ is an isomorphism if $i=0$ and $j \leq s, i=-1$ and $j \leq s-1$ or $i<-1$ and $i+j<s-i-2$.

PROOF. Let $\operatorname{Bar}^{* * *}\left(C_{1}\right)$ be the complex obtained from the bar resolution of $C_{1}$ as an $C_{1} \otimes C_{1}$-module and $\operatorname{Bar}^{* * *}\left(C_{2}\right)$ the similar complex constructed from $C_{2}$. Let $\alpha$ be an element of $\operatorname{Bar}^{-i . *}\left(C_{1}\right)$. We can write $u=a\left[b_{1}|\cdots| b_{i}\right] c$, where $a$ and $c$ are elements of $A$ and $b_{1}$ is an element of $\overline{C_{1} \otimes C_{1}}$. If there exists an element $b_{1}$ such that $\operatorname{deg} b_{l}>s$, then $\operatorname{deg} \alpha=\operatorname{deg} a+\operatorname{deg} b_{1}+\cdots+\operatorname{deg} b_{l}+\cdots+\operatorname{deg} b_{i}+\operatorname{deg} c-i$ 
$>0+2+\cdots+2+s+2+\cdots+2+0-i=s+i-2$ when $i \neq 0$. Thus we see that the morphism $\operatorname{Bar}^{i . j}(\theta): \operatorname{Bar}^{i . j}\left(C_{1}\right) \rightarrow \operatorname{Bar}^{i . j}\left(C_{2}\right)$, which is induced from $\theta$, is an isomorphism if $-i+j \leq s+i-2$ and $i \neq 0$. It is clear that $B^{0 . j}(\theta)$ is an isomorphism if $j \leq s$. Therefore we have Lemma 4.1.

PROOF OF THEOREM 1.3. Let $\left\{E_{r}, d_{r}\right\}$ be the Eilenberg-Moore spectral sequence converging to $H^{*}(L X ; \mathbf{Z} / p)$. By virtue of Proposition 3.3 and Lemma 4.1, we have a homomorphism $\psi$ from $B:=\Lambda\left(y_{1}, \ldots, y_{l}\right) \otimes \Gamma\left[\bar{y}_{1}, \ldots, \bar{y}_{l}\right] \otimes\left\{A_{p} /\left(d\left(\omega_{1}\right), \ldots\right.\right.$, $\left.\left.d\left(\omega_{m}\right)\right)_{A_{n}}\right\}$ to $E_{2}^{* * *}$ which is a monomorphism below degree 3. From [10, Proposition 4.2], it is seen that $\pi^{*}\left(y_{j}\right)=y_{j} \in F^{0} H^{*}(L X ; \mathbf{Z} / p)$ and $\pi^{*}\left(x_{i}\right)=x_{i} \in$ $F^{0} H^{*}(L X ; Z / p)$. Therefore the injectivity of $\pi^{*}$ allows us to conclude that $d_{2}$ : $E_{2}^{-2 . *} \rightarrow E_{2}^{0 . *}$ is trivial. Since $E_{2}^{i, j}=0$ if $q<-2 p$, it follows that the elements in $E_{2}^{-1 . j}$ survive in the $E_{\infty}$-term if $j \leq 4$. Thus we have an monomorphism $\psi: B^{\leq 3} \rightarrow\left(T \text { ot } E_{0}^{* * *}\right)^{\leq 3}$. In order to complete the proof of Proposition 1.3, we must solve extension problems below degree 3. More precisely, we need to consider whether it is true that $\sum \lambda_{i, j} x_{i} \bar{x}_{j}=0$ in $H^{*}(L X ; \mathbf{Z} / p)$ when $\sum \lambda_{i j} x_{i} \bar{x}_{j} \in \mathbf{Z} / p\left\{d\left(\omega_{1}\right), \ldots, d\left(\omega_{m}\right)\right\}$. Note that the element $\bar{x}_{j}$ in $E_{0}^{-1 . *}$ and its representative element in $H^{*}(L X ; \mathbf{Z} / p)$ are denoted by the same notation. Since the generators with degree 3 and filtration degree 0 are the elements $y_{1}, \ldots, y_{l}$, we can write the element $\sum \lambda_{i j} x_{i} \bar{x}_{j}$ as $\sum \mu_{k} y_{k}$ with some constants $\mu_{k}$. Let $\pi: L X \rightarrow X$ be the fibration defined by $\pi(\gamma)=\gamma(0)$. From [10, Proposition 4.2], we see that the element $y_{k}$ in $E_{0}^{0 . *}$ is identified with the element $\pi^{*}\left(y_{k}\right)$. Hence the given equality is written as $\sum \lambda_{i j} x_{i} \bar{x}_{j}=\sum \mu_{k} \pi^{*}\left(y_{k}\right)$. Let $s$ be the section of the fibration $L X \rightarrow X$ defined by $s(x)=C_{x}$, where $C_{x}$ is the constant loop at $x$. Since we can choose a representative element of $\bar{x}_{i}$ so that $s^{*}\left(\bar{x}_{i}\right)=0$ in $H^{*}(X ; Z / p)$, it follows that $\sum \mu_{k} y_{k}=s^{*}\left(\sum \lambda_{i j} x_{i} \bar{x}_{j}\right)=0$ in $H^{*}(X ; \mathbf{Z} / p)$ and hence $\mu_{k}=0$ for any $k$. Thus $\sum \lambda_{i, j} x_{i} \bar{x}_{j}=0$ in $H^{*}(L X ; \mathbf{Z} / p)$ when $\sum \lambda_{i j} x_{i} \bar{x}_{j} \in \mathbf{Z} / p\left\{d\left(\omega_{1}\right), \ldots, d\left(\omega_{m}\right)\right\}$. We have proved Theorem 1.3.

REMARK 4.2. In the case $p=2$, we cannot solve extension problems completely by using the usual argument on total degrees and column degrees of the associated bigraded algebra $E_{0}^{* * *}$. For example, it may be possible that $\bar{x}_{i}^{2}$ is equal to some $\bar{y}_{j}$. However some information about the squaring operations in $H^{*}(X ; \mathbf{Z} / 2)$ allows us to determine whether or not $\bar{x}_{i}^{2}$ is equal to $\bar{y}_{j}$. To be exact, if $S q^{1} x_{i}=\varepsilon y_{j}$ then $\bar{x}_{i}^{2}=\varepsilon \bar{y}_{j}$, where $\varepsilon=0$ or 1 . For details, see the proof of [6, Theorem 2.5].

PROOF OF THEOREM 1.4. Let $\left\{E_{r}, d_{r}\right\}$ be the Eilenberg-Moore spectral sequence used in the proof of Theorem 1.3. By applying [10, Proposition 4.5] and the same argument as the proof of [3, Lemma 1.3], we can show that $i^{*}\left(\bar{y}_{i}\right)=\sigma^{*}\left(y_{i}\right)$ and $i^{*}\left(\bar{x}_{j}\right)=\sigma^{*}\left(x_{j}\right)$ with any choice of representative elements of $\bar{y}_{i}$ and $\bar{x}_{j}$ in $E_{0}^{-1 . *}$. To proceed with the proof, we need the following lemma: 
LEMMA 4.3. For each indecomposable element $\bar{x}_{j}$ in $E_{0}^{-1 . *}$, one can choose its representative element $\bar{x}_{j}$ in $H^{*}(L X ; \mathbf{Z} / p)$ so that $\mathscr{D}_{X, p}\left(x_{j}\right)=\bar{x}_{j}$.

From Lemma 4.3 and the fact that the $D$-map $\mathscr{D}_{x . p}$ is a derivation, we can get $\mathscr{D}_{X, p}=\sum_{j=1}^{n} \bar{x}_{j} \partial / \partial x_{j}$. This completes the proof.

PROOF OF LEMMA 4.3. Let $f_{j}: X \rightarrow K_{j}=K\left(Z / p, n_{j}\right)$ be the map representing the element $x_{j}$ of $H^{n_{j}}(X ; \mathbf{Z} / p)$, where $n_{j}=\operatorname{deg} x_{j}$. From the naturality of the $D$-map $\mathscr{D}_{X . p}$, to prove Lemma 4.2, it suffices that $\mathscr{D}_{K_{j}, p}\left(\iota_{j}\right)=\bar{\iota}_{i}$, where $\iota_{j}$ is the fundamental element of $H^{n_{j}}\left(K_{j} ; \mathbf{Z} / p\right)$ and $\bar{\iota}_{j}$ is the element corresponding to $\iota_{j}$ (see Theorem 1.3). We denote the map $\int_{S^{1}} \circ e v^{*}: H^{*}\left(K_{j} ; \mathbf{Z} / p\right) \rightarrow H^{*}\left(S^{1} \times \Omega K_{j} ; \mathbf{Z} / p\right) \rightarrow$ $H^{*-1}\left(\Omega K_{j} ; Z / p\right)$ by $\tilde{D}$. Here $\Omega X$ means the space of continuous loops on $X$ which map $1 \in S^{\prime}$ to the base point of $X$. Let $\sigma^{*}: H^{*}\left(K_{j} ; \mathbf{Z} / p\right) \rightarrow H^{*-1}\left(\Omega K_{j} ; \mathbf{Z} / p\right)$ be the cohomology suspension. Since $i^{*}\left(\bar{l}_{j}\right)=\sigma^{*}\left(l_{j}\right)$ and $\sigma^{*}\left(l_{j}\right)$ is the fundamental element in $H^{n_{j}-1}\left(\Omega K_{j} ; \mathbf{Z} / p\right)=H^{n_{j}-1}\left(K\left(\mathbf{Z} / p, n_{j}-1\right) ; \mathbf{Z} / p\right)$, we see that if $\tilde{\mathscr{D}}\left(\iota_{j}\right)=\sigma^{*}\left(\iota_{j}\right)$ then $\mathscr{D}_{K_{j}, p}\left(\iota_{j}\right)=\bar{\iota}_{j}$. We now prove $\tilde{\mathscr{D}}\left(\iota_{j}\right)=\sigma^{*}\left(\iota_{j}\right)$. Let $f:\left(I^{n_{j}}, \partial I^{n_{j}}\right) \rightarrow\left(K_{j}, *\right)$ be a continuous map and $g: I^{n_{j}-1} \rightarrow \Omega K_{j}$ a map defined by $g(t)(s)=f(t, s)$ for $t \in I^{n_{j}-1}$ and $s \in I$. The argument of the proof of [8, Proposition 2.1] enables us to deduce that the element dual $[f]$ in $\operatorname{dual}\left(\pi_{n_{j}}\left(K_{j}\right) \otimes \mathbf{Z} / p\right)$ is mapped to dual $[g]$ in dual $\left(\pi_{n_{j}-1}\left(\Omega K_{j}\right) \otimes \mathbf{Z} / p\right)$ by $\tilde{\mathscr{D}}$ under the isomorphisms $H^{n_{j}}\left(K_{j} ; \mathbf{Z} / p\right) \cong \operatorname{dual}\left(H_{n_{j}}\left(K_{j} ; \mathbf{Z} / p\right)\right) \cong \operatorname{dual}\left(\pi_{n_{j}}\left(K_{j}\right) \otimes \mathbf{Z} / p\right)$ and $H^{n_{j}-1}\left(\Omega K_{j} ; \mathbf{Z} / p\right) \cong \operatorname{dual}\left(\pi_{n_{j}-1}\left(\Omega K_{j}\right) \otimes \mathbf{Z} / p\right)$. Let $\varepsilon_{1}: P K_{j} \rightarrow K_{j}$ be the path-loop fibration defined by $\varepsilon_{1}(\gamma)=\gamma(1)$. Then the homology suspension $H_{n_{j}-1}\left(\Omega K_{j} ; Z\right) \stackrel{\cong}{\longleftarrow}$ $H_{n_{j}}\left(P K_{j}, \Omega K_{j} ; \mathbf{Z}\right) \stackrel{\varepsilon_{*} ;}{\rightarrow} H_{n_{j}}\left(K_{j} ; \mathbf{Z}\right)$ is regarded as the homomorphism $\pi_{n_{j}-1}\left(\Omega K_{j}\right) \stackrel{\cong}{\rightleftarrows}$ $\pi_{n_{j}}\left(P K_{j}, \Omega K_{j}\right) \stackrel{\varepsilon_{1}}{\rightarrow} \pi_{n_{j}}\left(K_{j}\right)$ under the identification with the Hurewicz maps. We define the map $\tilde{f}:\left(I^{n_{j}}, \partial I^{n_{j}}, I^{n_{j}-1} \times 0 \cup \partial I^{n_{j}-1} \times I\right) \rightarrow\left(P K_{j}, \Omega K_{j}, *\right)$ by $\tilde{f}(t, s)(u)=$ $*$ if $0 \leq u \leq 1 /(s+1)$ and $\tilde{f}(t, s)(u)=f(t, u(s+1)-1)$ if $1 /(s+1) \leq u \leq 1$. Then we can deduce that $\varepsilon_{1 *}(\tilde{f})=f$ and that $\partial(\tilde{f})$ is homotopic to $g$ by the homotopy $H:\left(I^{n_{j}-1} \times I, \partial I^{n_{j}-1} \times I\right) \rightarrow\left(\Omega K_{j}, *\right)$ defined by $H(t, l)(u)=*$ if $0 \leq u \leq l / 2$ and $H(t, l)(u)=f(t,(2 u-l) /(2-l))$ if $l / 2 \leq u \leq 1$. Thus it follows that cohomology suspension $\sigma^{*}$ maps dual $[f]$ to $\operatorname{dual}[g]$. This completes the proof.

We can determine the structure of the kernel of the $D$-map $\mathscr{D}_{X . p}: H^{4}(X ; \mathbf{Z} / p) \rightarrow$ $H^{3}(L X ; \mathbf{Z} / p)$ completely. Let $\eta_{p}: H^{4}(X ; \mathbf{Z}) \rightarrow H^{4}(X ; \mathbf{Z} / p)$ be the $\bmod p$ reduction. Then we have

PROPOSITION 4.4. Suppose that $X$ is a simply connected space and that there exists a morphism of algebras $\psi$ to $H^{*}(X ; \mathbf{Z} / p)$ from an algebra $\Lambda\left(y_{1}, \ldots, y_{l}\right) \otimes$ $\mathbf{Z} / p\left[x_{1}, \ldots, x_{n}\right] /\left(\rho_{1}, \ldots, \rho_{m}\right)$ which is an isomorphism below degree 4 , where $\operatorname{deg} \rho_{i}$ $=4$. If $x$ is an element in the kernel of the D-map $\mathscr{D}_{X}=\int_{S^{1}} \circ e v^{*}: H^{4}(X ; \mathbf{Z}) \rightarrow$ $H^{3}(L X ; \mathbf{Z})$, then $\eta_{2}(x)=\sum \lambda_{i} x_{i}^{2}$ for some constant $\lambda_{i}$ and $\eta_{p}(x)=0$ if $p \neq 2$. 
PROOF. By virtue of Theorem 1.3 and Theorem 1.4, we see that $\mathscr{D}_{X . p}$ coincides with the operator $\sum_{i=1}^{n} \bar{x}_{i} \partial / \partial x_{i}$. Therefore, the image of the map $\mathscr{D}_{x . p}$ is included in the image of $\psi$ of Theorem 1.3. Hence, we can deduce that if $\mathscr{D}_{X . p}(\alpha)=0$ for some $\alpha \in H^{4}(X ; \mathbf{Z} / p)$, then

$$
\sum_{i=1}^{n} \frac{\partial \alpha}{\partial x_{i}} \bar{x}_{i} \in\left(d\left(\omega_{1}\right), \ldots, d\left(\omega_{m}\right)\right)_{A_{p}}
$$

We can write $\alpha=\alpha_{1}+\alpha_{2}$ by using elements $\alpha_{1}$ and $\alpha_{2}$ which are linear combinations of $x_{i} x_{j}$ and $x_{k}$ respectively. From the definition of $d\left(\omega_{j}\right)$, it follows that $\alpha_{2}=0$ and

$$
\sum_{i=1}^{n} \frac{\partial}{\partial x_{i}} \bar{x}_{i}\left(\alpha_{1}-\sum \xi_{j} \rho_{j}\right)=0
$$

in $A_{p}$ for some $\xi_{j}$ in $Z / p$. Since $\operatorname{deg} \rho_{i}=4$ and $\operatorname{deg} \partial\left(\alpha_{1}-\sum \xi_{j} \rho_{j}\right) / \partial x_{i}=2$ for any element $x_{i}$ with degree 2 , one can conclude that $\partial\left(\alpha_{1}-\sum \xi_{j} \rho_{j}\right) / \partial x_{i}=0$ in $\mathbf{Z} / p\left[x_{1}, \ldots, x_{n}\right]$ for any $i$. Thus, in $\mathbf{Z} / p\left[x_{1}, \ldots, x_{n}\right] /\left(\rho_{1}, \ldots, \rho_{m}\right), \alpha_{1}=0$ if $p \neq 2$ and $\alpha_{1}=\sum \lambda_{i} x_{i}^{2}$ if $p=2$.

PROOF OF THEOREM 1.1. For any element $x$ in $\operatorname{Ker}\left\{\mathscr{D}_{M}: H^{4}(X ; \mathbf{Z}) \rightarrow\right.$ $\left.H^{3}(L X ; Z)\right\}$, its $\bmod p$ reduction $\eta_{p}(x)$ is zero if $p \neq 2$ by Proposition 4.4. Therefore it follows from (1.1) that the free part and odd torsion part of $x$ is zero. Moreover, the condition (1.2) enables us to deduce that the 2-torsion part of $x$ is zero.

REMARK 4.5. So far, we have considered the string class of an $S O(n)$-bundle in the case where $n \geq 5$. The case $n=4$ must be treated separately as mentioned in [8, Remark, page 150] because the universal central extension of $L \operatorname{Spin}(n)$ is an extension by a 2-torus. The fact that $S O(4)$ is not simple causes the difference. In the case where $n=3$, since $S O(3)$ is simple, we can define the string class of an $S O$ (3)-bundle with a spin structure in similar fashion to the case $n \geq 5$. However, the index of the homomorphism $B \pi^{*}: H^{4}(B S O(3) ; \mathbf{Z})=\mathbf{Z} \rightarrow H^{4}(B S p i n(3) ; \mathbf{Z})=\mathbf{Z}$ is 4 , not 2 , where $\pi: \operatorname{Spin}(3) \rightarrow S O(3)$ is the universal covering. This fact is proved by using the same argument as the proof of [8, Lemma 2.2, page 148]. Notice that $H^{5}(B S O(3) ; \mathbf{Z})$ is zero though $H^{5}(B S O(n) ; \mathbf{Z})=\mathbf{Z} / 2$ for $n \geq 5$. Thus the string class $\mu(Q)$ of an $S O$ (3)-bundle $\xi$ with a spin structure $Q \rightarrow M$ can be regarded as the image of $1 / 4$ the Pontrjagin class of $\xi$ by the $D$-map $\mathscr{D}_{M}: H^{4}(M ; Z) \rightarrow H^{3}(L M ; Z)$. 


\section{References}

[1] D. Burghelea and M. Vigué-Poirrier, Cyclic homology of commutative algebras I, Lecture Notes Math. 1318 (Springer, New York, 1990).

[2] E. B. Dynkin, 'Semisimple subalgebras of semisimple Lie algebras', Mat. Sb. 30 (1952), 349-462 [Russian], Amer. Math. Soc. Transl. 6 (1957), 111-244.

[3] K. Kuribayashi, 'On the mod $p$ cohomology of spaces of free loops on the Grassmann and Stiefel manifolds', J. Math. Soc. Japan 43 (1991), 331-346.

[4] - 'On the vanishing problem of string classes', J. Austral. Math. Soc. (Series A) 61 (1996), 258-266.

[5] - 'Module derivations and the adjoint action of finite loop space', preprint Okayama University of Science, 1998.

[6] K. Kuribayashi and T. Yamaguchi, 'The cohomology algebra of certain free loop spaces', Fund. Math. 154 (1997), 57-73.

[7] J. McCleary and W. Ziller, 'On the free loop space of homogeneous spaces', Amer. J. Math. 109 (1987), 765-782. Correction: Amer. J. Math. 113 (1991), 375-377.

[8] D. A. McLaughlin, 'Orientation and string structures on loop space', Pacific J. Math. 155 (1992), 143-156.

[9] A. L. Oniscik, 'Transitive compact transformation groups', Mat. Sb. 60 (1663), 447-485 [Russian], Amer. Math. Soc. Transl. 55 (1966), 153-194.

[10] L. Smith, 'Homological algebra and the Eilenberg-Moore spectral sequence', Trans. Amer. Math. Soc. 129 (1967), 58-93

[11] - 'On the characteristic zero cohomology of the free loop space', Amer. J. Math. 103 (1981), 887-910.

Department of Applied Mathematics Graduate School of Science and Technology Okayama University of Science Okayama University 1-1 Ridai-cho, Okayama 700 Okayama 700 Japan Japan e-mail: kuri@geom.xmath.ous.ac.jp e-mail: t_yama@math.okayama-u.ac.jp 